\title{
A Design for Large-Area Fast Photo-detectors with Transmission-Line Readout and Waveform Sampling
}

\author{
B. Adams, J. T.Anderson, K. Attenkofer, M. Bogdan, Member IEEE, K. Byrum, \\ G. Drake, Member IEEE, J. Efflam, H. J. Frisch, J-F. C. Genat, Member IEEE, M. K. Heintz, \\ Z. Insepov, V. Ivanov, E. N. May, T. Natoli, K. Nishimura, R. Northrop, A. Paramonov, M. Pellin, \\ E. Ramberg, A. Ronzhin, D. Routkevitch, L. Ruckman, M. Sanchez, G. Sellberg, O. Siegmund, \\ R. Stanek, F. Tang, Member IEEE, A. Tremsin, Member IEEE, G. Varner, Member IEEE, J. Va'Vra, \\ H. H. Wang, H. Weerts, M. Wetstein, T. Zhao, A. Zinoviev.
}

\begin{abstract}
-
We present a preliminary design and the results of simulation for a photo-detector module to be used in applications requiring the coverage of areas of many square meters with time resolutions less than 10 picoseconds and position resolutions of less than a millimeter for charged particles. The source of light is Cherenkov light in a radiator/window; the amplification is provided by panels of micro-pores functionalized to act as microchannel plates (MCPs). The good time and position resolution stems from the use of an array of parallel $50 \Omega$ transmission lines (strips) as the collecting anodes. The anode strips feed multi-GS/sec sampling chips which
\end{abstract}

Manuscript received June 14th, 2009. This work was supported in part by the University of Chicago, the National Science Foundation under grant number 5-43270, the U.S. Department of Energy Advanced Detector Research Program, and UChicago Argonne, LLC, Operator of Argonne National Laboratory ("Argonne"). Argonne, a U.S. Department of Energy Office of Science laboratory, is operated under Contract No. DEAC0206CH11357.

Corresponding author is J-F. Genat. e-mail: genat@ hep.uchicago.edu.

B. Adams, J.T. Anderson, K. Attenkofer, K. Byrum, G. Drake, J. Efflam, Z. Insepov, E. N. May, A. Paramonov, M. Pellin, M. Sanchez, R. Stanek, H.H. Wang, H. Weerts, and M. Wetstein are with Argonne, Argonne IL, 60439 USA,

M. Bogdan, H. J. Frisch, J-F. Genat, M. K. Heintz, T. Natoli, R. Northrop,

F. Tang are with the Enrico Fermi Institute at the University of Chicago, 5640 S. Ellis Av, Chicago IL, 60637 USA,

V. Ivanov is with Muons Inc., 552 N. Batavia Avenue, Batavia, IL 60510 USA,

A. Zinoviev is with Arradiance, 142 North Road, Suite F-150, Sudbury, MA 01776 USA

E. Ramberg, A. Ronzhin, and G. Sellberg, are with the Fermi National Laboratory, PO Box 500, Batavia, IL 60510 USA,

K. Nishimura, L. Ruckman and G. Varner are with the University of Hawaii, 2505 Correa Road, Honolulu, HI 96822 USA,

J. Va'Vra is with the Stanford Linear Accelerator Center, Menlo Park, CA 94025 USA,

O. Siegmund and A. Tremsin are with the Space Sciences Laboratory, University of California, One Cyclotron Road, Berkeley 94720 USA,

D. Routkevitch is with Synkera Technologies, 2021 Miller Drive, Suite B, Longmont, CO 80501 USA,

T. Zhao is with the University of Washington, Seattle, WA 98195 USA. digitize the pulse waveform at each end of the strip, allowing a measurement of the time from the average of the two ends, and a 2-dimensional position measurement from the difference of times on a strip, and, in the orthogonal direction, the strip number, or a centroid of the charges deposited on adjacent strips. The module design is constructed so that large areas can be 'tiled' by an array of modules

\section{INTRODUCTION}

T arge-area robust cheap photo-detectors would be transformational in a wide variety of areas. Possible applications include cheaper and more precise Positron Emission Tomography (PET) cameras in medical imaging, scanners for transportation security, and particle detectors in high-energy neutrino and collider physics, astrophysics, and nuclear physics. There would also be many possibilities for new products and spin-off technologies. Because the new devices would be planar, relatively thin, and physically robust, they would require less volume and infrastructure in large-area applications for which photomultipliers are presently the current solution.

The goal is to develop a family of large-area robust photodetectors that can be tailored for a wide variety of applications for which large-area economical photon detection would be transformational. Progress in modern micro-electronics, materials science, and nano-technology gives us an opportunity to apply micro-channel plate technology to produce large-area robust photo-detectors with excellent space and time resolution. In addition to having excellent resolution, the new devices should be relatively economical to produce in quantity, being a simple sandwich of layers rather than an assembly of discrete parts. There would be additional economies associated with the overall system design for applications such as large-area detectors, precision time-offlight measurements at particle accelerators such as the LHC, RHIC, JPARC, Super-B, and the ILC; Positron-Emission 
Tomography; large-area detectors such as those being proposed for DUSEL; and non-proliferation security.

The development of high efficiency photo-cathodes, based on nano-sciences technologies such as Atomic Layer Deposition (ALD) [1-2], Anodic Aluminum Oxide by anodization (AAO) [3], or recent glass technologies [4] would allow implementing large area detectors at possibly cheap assembly techniques. We have already demonstrated [5-6] that fast waveform sampling using CMOS ASICs at both ends of transmission line anodes allows the coverage of large areas with small numbers of channels, permitting excellent time and space resolution and a built-in noise identification and reduction mechanism.

The paper is organized as follows: The introduction has given a brief introduction to the motivation for considering micro-channel plate technology for large-area photo-detectors, and briefly summarized several of the applications in science and medicine for which the technology would be transformational. The first section introduces the photocathode problem, the second section presents two possible implementations of the pore's amplification, and the third section is devoted to the charge collection and digitization. The mechanical design is then introduced, as well as system aspects and proposed designs in the last section.

\section{Motivation}

Photomultipliers are truly remarkable devices, loved and admired by anyone who has worked with them. They are highbandwidth, high-gain, current-source amplifiers, with high quantum efficiencies for photons (QE) [7] and the ability to resolve single photo-electrons. Photomultipliers (PMT's) can have excellent time resolution [8], and multi-anode PMT's allow spatial resolution as well [9]. Specialized PMTs and hybrid variations can be tailored for specific high precision use [10-11]. In addition, PMTs are robust, well-understood, easy to use, and can be quite inexpensive.

Micro-channel plate photo-multipliers (MCP-PMT) are an evolution from the basic principles of photomultipliers, and have been developed to be a robust and mature technology [12-17]. These carefully crafted planes of small pores, which form the amplification sections of the complete MCP-PMT device, have been thoroughly characterized [18-21], and sophisticated simulation programs and an extensive literature exist to predict performance [21-23] Micro-channel plate photomultipliers combine the virtues of photo-multipliers with a relatively simple planar package construction that lends itself to scaling to large-area detectors. In addition, MCPPMT's employ a local amplification construction with very small path-lengths for the photon conversion and electron multiplication, resulting in exceptionally fast rise times [24].

Initially driven by the desire to do particle identification at colliders, we have asked the question whether can one make a photo-device that has all these characteristics at once, in particular both large area and good time resolution. In the process, we have solved one of the fundamental problems: the complementarity between time resolution and large transverse dimensions. Two other fundamental problems remain largearea photo-cathodes and batch-produced amplification sections, as discussed below. However, we believe that there are no "show-stoppers" for either of these, and a concerted attempt will solve these as well. The solution to the first problem, which has allowed us to move to the next two, is an anode design that uses transmission lines digitized on both ends by waveform sampling to get position (from the difference in times on the two ends) and time (from the average). The solution is economical as electronics goes as L, the transverse size of the MCP-PMT, rather than $\mathrm{L}^{2}$, as would a pixel detector. The solution also scales well to large transverse sizes, up to many feet in a single panel. There is extensive successful experience with wave-form sampling integrated circuits [25-28]; sampling chips exist now for many applications, and work is now underway to develop designs in silicon processes with smaller feature size capable of reaching resolutions of a few pico-seconds [30]. Some applications motivating the proposal are briefly listed below, with more detail given in [30].

\section{TOF systems.}

High-precision time-of-flight systems, with resolutions below a few pico-seconds, for new or upgraded detectors in particle and nuclear physics. Such a system would provide photon vertexing, multiple-interaction separation, and chargeparticle identification. Possible candidates are the $\mathrm{LHCb}$ upgrade, the ATLAS upgrade, ALICE, Super-B, future detectors at a muon collider or ILC, and experiments at the precision frontier such as $\mathrm{K}_{1}-\pi^{0} \mathrm{v} \mathrm{v}_{\mathrm{bar}}$ at J-PARC.

\section{- $\quad$ Positron Emission Tomography (PET).}

Utilizing the thin planar geometry of these photo-detectors to construct a Positron Emission Tomography (PET) camera using thin crystals in a sampling-calorimeter configuration, giving depth-of-interaction information as well as superb energy and time resolution. In addition this design would cut system costsas well as the cost of the photo-devices, as the crystal preparation cost would be much reduced, resulting in a highly economical all-digital design of a clinical PET scanner with depth-of-interaction and TOF measurement [31-36].

- $\quad$ Large water Cherenkov detectors.

A large water Cherenkov detector with active photon coverage over essentially $100 \%$ of the surface area, using large-area planar MCP-PMTs [37]. Photo-detectors with complete coverage and 100-ps-time and 1-inch position resolutions on each photon may allow new capabilities, such as increased fiducial volume, $\pi_{0}-\mathrm{e}^{-}$separation, track reconstruction, and possibly even the introduction of a low magnetic field for charge determination.

\section{- Diagnostics at accelerators.}

Diagnostics at accelerators and in beam lines, such as the 
MANX muon cooling experiment, or the Advanced Photon Source (APS) at Argonne.

\section{- Security}

Non-proliferation and transportation security. MCP's can be optimized for neutron or photon detection; large area panels would allow economical scanners for containers and trucks.

The different applications have different cost targets, as the areas covered and resolution requirements are so different. For example, the surface area of the typical collider solenoid to be tiled by MCP-PMT's [38], or of a precision time-of-flight (TOF) plane at $\mathrm{LHCb}[38]$, is $30 \mathrm{~m}^{2}$. Similarly, for Positron Emission Tomography, one planar 8 inch $\mathrm{x} 8$ inch MCP would typically replace 64 photo-tubes and bases, with additional reduced electronics costs, in an order-of-magnitude lower photo-device cost as well as increased capability.

For use in a large water Cherenkov detector, to economically achieve 'hermetic' (close to $100 \%$ coverage), which may allow sub-radiation-length vertex resolution for $\pi^{0}-\mathrm{e}^{-}$separation and tracking capability requires the ability to integrate the individual components into large panels.

One should notice that resolution is under $10 \mathrm{ps}$ for charged particles and photon-vertexing to $3 \mathrm{~mm}$ in the direction of flight. Simulation studies of the effects of light-scattering and multiple scattering of the charged particles on these tracking capabilities are beginning inside the DUSEL collaboration with joint members of our group. For a notable example, see [1].

\section{THE "FrUGAL" MCP.}

Up to this point we have done our development R\&D using commercial MCP-PMTs, which are typically a factor of 10 to 100 more expensive per unit area than a typical PMT [12]. However, it has been an instructive exercise to consider a 'strawman' design for a simple MCP-PMT, to be used as a test-bed for scaling up the interior pieces to larger areas. Figure 1 shows a 'cartoon' of the concept of an economical large-area proto typical MCP-PMT module, which in principle could be the basis for a much larger mosaic of either complete module or only the active layers in a single common shell (4foot-long anode transmission lines that span multiple 8-inch panels will a bandwidth over $1 \mathrm{GHz}$ ). We are exploring the costs for scaling up a conventional ceramic body, used with immense success for many years at SSL [39-41] and industry, and also for an all-glass design, for which there exists an industrial base for televisions, monitors, and other displays [41], and also extensive expertise at Argonne. The glass alternative is attractive in principle for size and cost MCPPMT production already solved in the ceramic solution: applying transmission lines to the anode plane, internal electrical connections, getting the signals out without too much loss of fidelity, glass-to-glass seals, mechanical integrity, implementation of getters, and bake-out, among others.

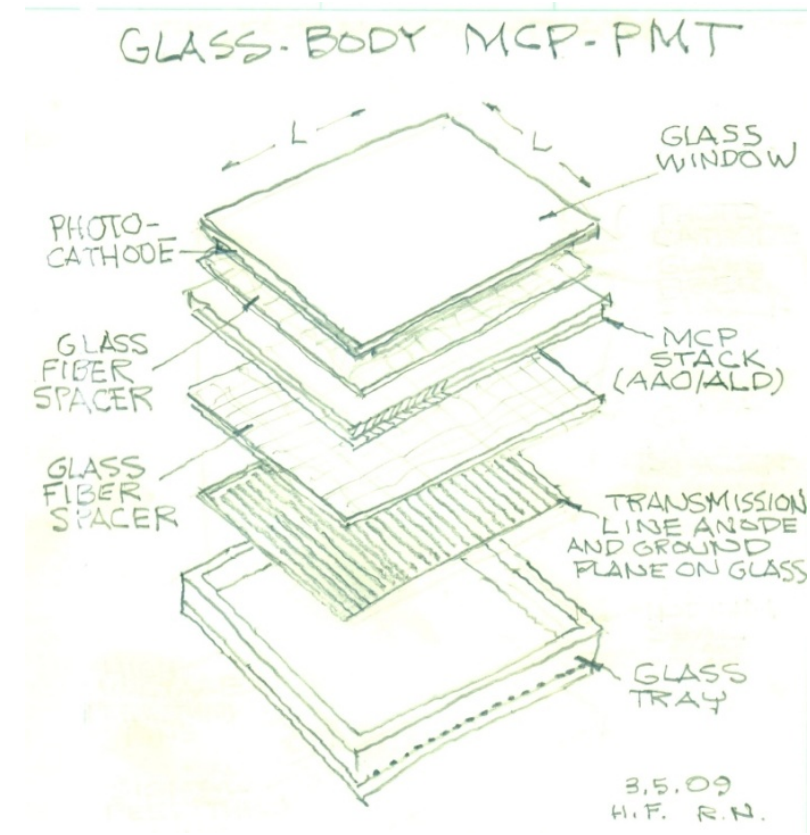

Figure 1. A 'cartoon' of the concept of a glass 'frugal' MCP-PMT, used as the basis for discussion of design choices and costs. The goal is to reduce the cost of each of the components and of the overall assembly. We are considering construction both with ceramic, for which there is extensive experience [15-17] and glass, such is used in flat-panel televisions. The interior pieces lend themselves to 'tiling' large areas within a panel, and would be integrated into a single monolithic detector sub-assembly. The development of a robust nano-structured photo-cathode material would allow by-passing vacuum assembly, making the assembly of large panels feasible. Note that the pattern and layout of the anode transmission line is easily optimized to the desired granularity and geometry, with the number of channels variable from one to many.

We see three areas that are critical to achieving economical large-area MCP-PMT's. They are:

- The photo-cathodes

The scale-up and modification of photo-cathode production technology for larger area planar devices [42-43].

- The first strike problem

Implementing solutions to the 'first-strike' problem of developing substrates that satisfy the geometry for channelplate amplification, are low cost and scalable to large areas, and that may also allow new geometries with higher photon detection efficiency and improved single photo-electron resolution:

- Solving the time/amplitude/transverse-size complementarity problem.

We have spent the last several years solving the third problem, and the solution, transmission-line anodes to preserve the signal amplitude and shape with cheap fast lowpower waveform sampling on both ends, is the breakthrough that enables the creation of large-area photo-detectors $[5,6,46]$, 
The first two problems remain to be solved.

\section{Photo-CATHODES.}

\section{A. Conventional Bialkali Photo-cathodes}

There have been major advances in photo-cathode development in the recent years [1]. Figure 2 shows the recent gains in quantum efficiency by Hamamatsu versus wavelength (left-hand panel), as well as the spectrum of Cherenkov light as observed through water [45] as a reference input spectrum. The technique is very advanced, giving quantum efficiencies of over $40 \%$ in the UV/visible spectral regions today. In addition, photocathode processing is low cost and has good yields [46].

We plan to use conventional bialkali photo-cathodes in an $800 \times 800$ format and a conventional transmission geometry as the primary choice. SSL has a long history of successfully producing photo-cathodes [39-42].

The SSL group has produced conventional photo-cathodes larger than 5 -inch ${ }^{2}$, and has the facilities to go to 8 -inch square, the size we are considering as our basic sub-assembly. The 8-inch size will also fit in process equipment routinely used by the semi-conductor industry.

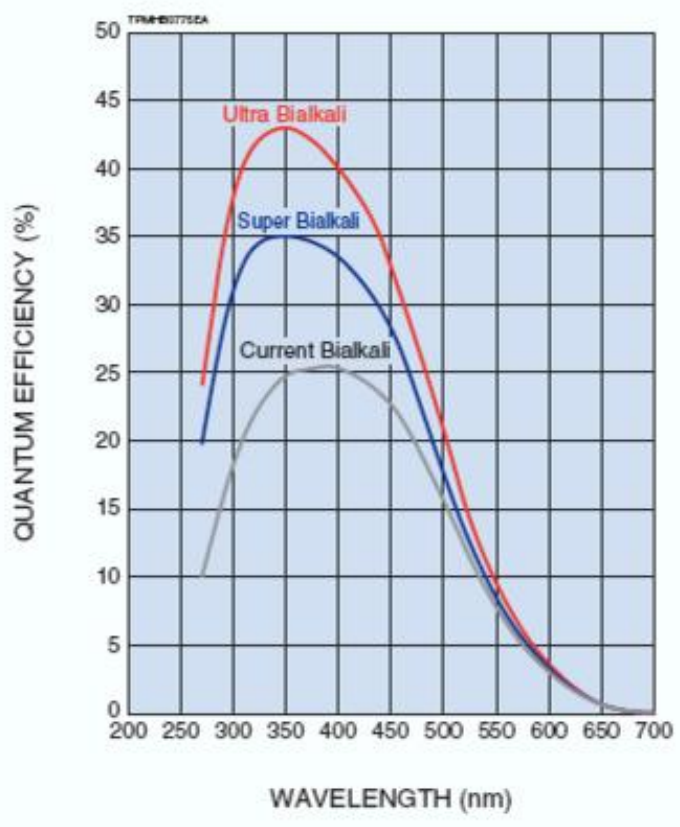

Figure 2a. Quantum efficiencies for Hamamatsu bialkali photo-cathodes, showing the recent developments.

\section{B. $R \& D$ on Nano-scale Photo-cathodes}

In parallel with getting bialkali quantum-efficiency up to industry standards, an effort will develop advanced photocathodes using the facilities and strengths available in nanomaterials at Argonne.

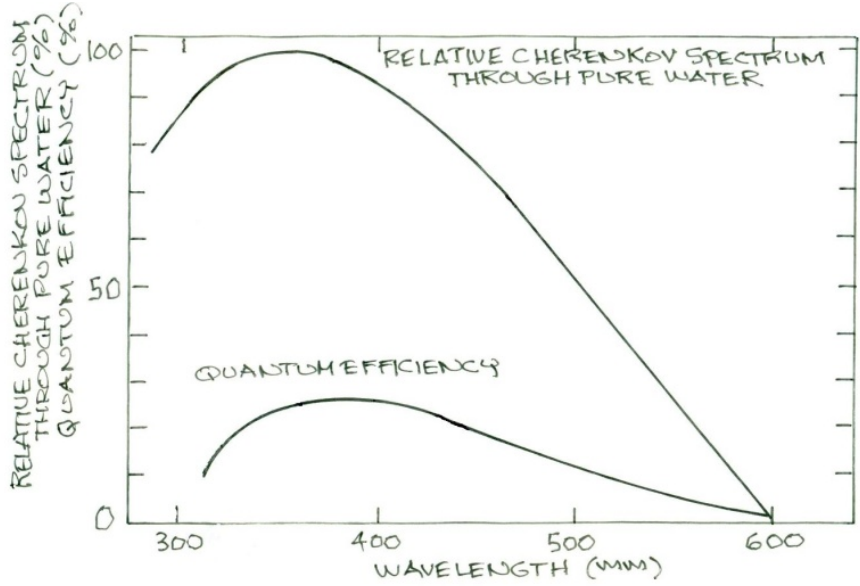

Figure $2 \mathrm{~b}$ The spectrum of Cherenkov light in water, and the quantum efficiency of the Hamamatsu 20-inch photo-tube used in Super-K (from [47])

The effort will share characterization and test facilities, standard substrates, standard measurement criteria, and expertise with the mainstream effort on bialkali photocathodes. In particular, the goal is to develop novel photocathodes that appear "black" to photons in the required spectral region, have high electron emissivity, and can be applied to large panels using Atomic Layer Deposition (ALD) or Chemical Vapor Deposition (CVD) [43].

Expertise will be needed on the physics of photo-cathodes, the material fabrication and processing of the photo-cathodes, surface and material properties of the photocathode using various techniques such as x-ray diffraction and photoemission, and the characterization of the photoelectrons generated from these photo-cathodes, such as the distributions in energy and angle. This will allow applying the revolution in surface chemistry due to the application of sophisticated surface analysis facilities and theoretical knowledge implemented in simulations to large-area MCP-PMT's. Advanced characterization of efficiency versus composition using several highly-evolved facilities at Argonne will be performed.

\section{Characterization facilities.}

For characterization we will need structural and electronic probes, an automated fast test capability, local probes for work-function measurements, and the ability to make timing and emission angle measurements. For simulation we will need both static and dynamical models and band-structure calculations. We have access to the Center for Nanoscale Materials at Argonne for initial production.

\section{AMPLIFICATION}

The second problem on the critical path is to make arrays of channels that have the required characteristics for high efficiency and gain, low noise, a good transit-time-spread, and good amplitude resolution for single photo-electrons. These characteristics include providing a well-defined surface for the 
first-strike for the incoming photo-electron, large open-arearatios, and an interior surface with electrical and physical properties to sustain a cascade with enough gain and current capability, while maintaining an acceptable dark current.

\section{A. Int roduction to Amplification in Micro-Channels.}

Figure 3a shows the basics of channel geometry. First, the channel needs to provide a first strike for the incoming photoelectrons, followed by successive strikes which provide the amplification. The surface of the channel must provide a current-carrying layer that provides adequate charge, much as the dynode string in a photo-multiplier base does, and a functionalized complex surface layer that provides gain for each strike [43].

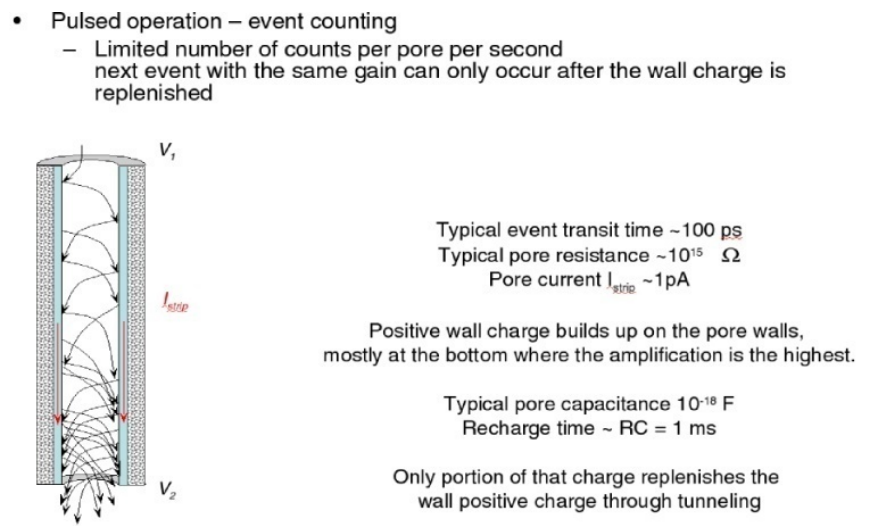

Figure 3a. The basics of amplification in a channel. The underlying currentcarrying layer provides charge for the shower, and the functionalized complex surface layer provides gain for each strike.
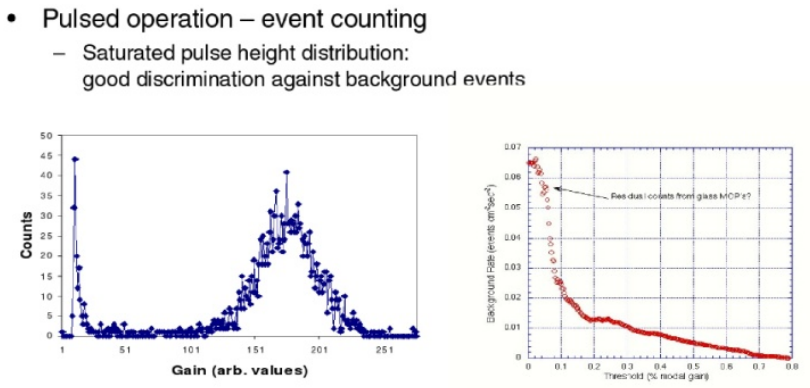

Typical pulse height distribution GALEX satellite detector

Background rate of Si MCPS
as a function of threshold

Figure 3b. A typical pulse height distribution and the rate from a glass MCP versus threshold showing the good separation of background in saturated mode. (from A. Tremsin [31]).

An example of the studies we are doing on the details of the showering inside the channel, with the goal of optimizing transit-time-spread, gain variations, and saturation [46] However, these simulations are only as good as the input data on the materials, and so a parallel program to survey existing data has started. The emphasis on characterization in our group using the Argonne facilities is driven by this need to have input data that we can trust [47]
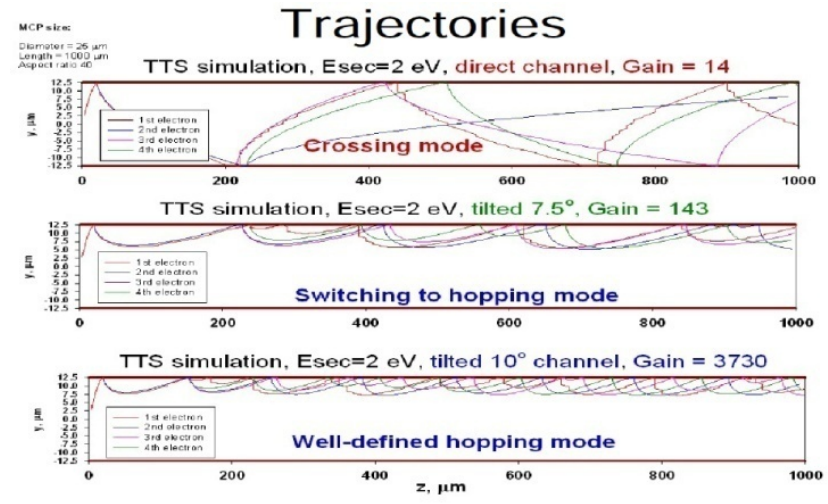

Figure 4. A slide from a study of transit-time-spread in pores (Z. Insepov and $\mathrm{V}$. Ivanov), demonstrating the power of simulation $\backslash$ cite $\{$ field $\}$. This also demonstrates how critical it is to have correct input data characterizing the properties of materials

\section{B. MCP's with Atomic Layer Deposition.}

The availability of the technique of Atomic Layer Deposition, ALD, to functionalize MCP's revolutionizes the possibilities for MCP construction by separating the functions of the substrate, which provides the channel geometry, and the ALD surface layers, which provide the current and gain. The use of ALD for MCP's has been proven over the last several years [21], and a great deal of work has been done understanding the basic issues and exploring the multidimensional parameter space $[48,1,2,9,49,50,21]$, gives more detail on the use of ALD for MCP's. ALD also gives the possibility of eventually functionalizing the channels so that they have an internal structure similar to that of a photomultiplier, with well defined first and second dynodes, for example, in this case implemented as bands of enhanced secondary emission [46]. It may be possible to use ALD to make a photocathode in an 'opaque' geometry on the surface of a funnel around the channel opening, as shown in Figure 10, giving a large open-area-ratio even with small channels. Since most of the time jitter is introduced in the photon conversion and photo-electron drift and the first several strikes in the amplification process, this geometry, with its tight control over the shower development, is predicted to have a remarkable time resolution.

In addition, defining the geometry of the shower evolution, much as it is defined by the discrete dynodes of a photomultiplier, should improve the single photo-electron peak resolution (this is one of the areas we will be addressing using the Muons Inc. MCP simulation).

Figure 5 shows curves of output current versus input current for an MCP functionalized with ALD [50] and a model that is in good agreement with observations. We start with access to this experience inside the collaboration, with channel performance better than with the standard commercial MCP's [50]. 
Current amplification mode Output current is limited by the MCP resistance

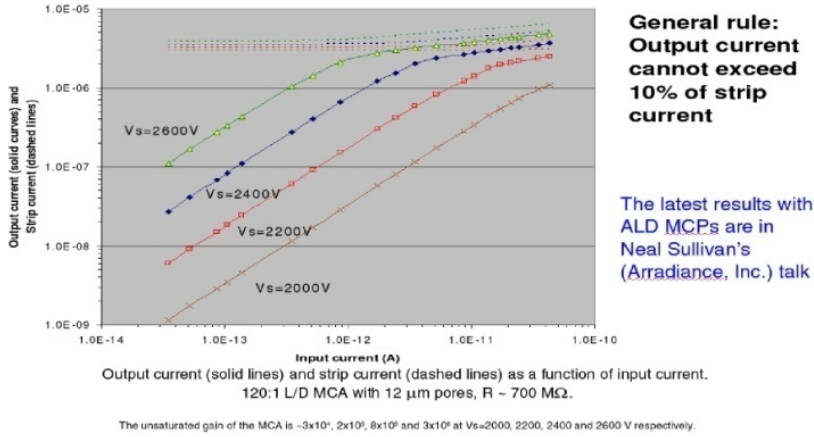

Figure 5. Curves of output current vs input current for an MCP functionalized with ALD

At the same time, with the combined expertise in characterization and ALD of the group it is possible that we can find major improvements in gain and effective collection area that will translate directly into coverage.

\section{Voltage along the pore vs. saturation}

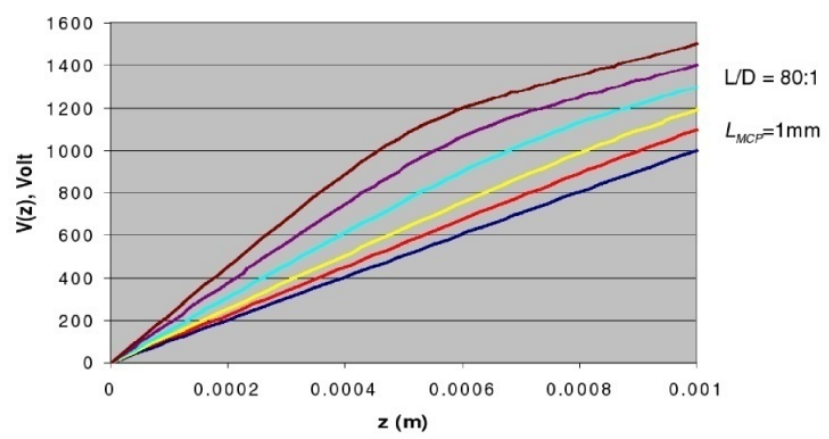

Figure 6a. The predicted electric field distribution along the channel for different operating voltages.

\section{E-field along the pore vs. saturation}

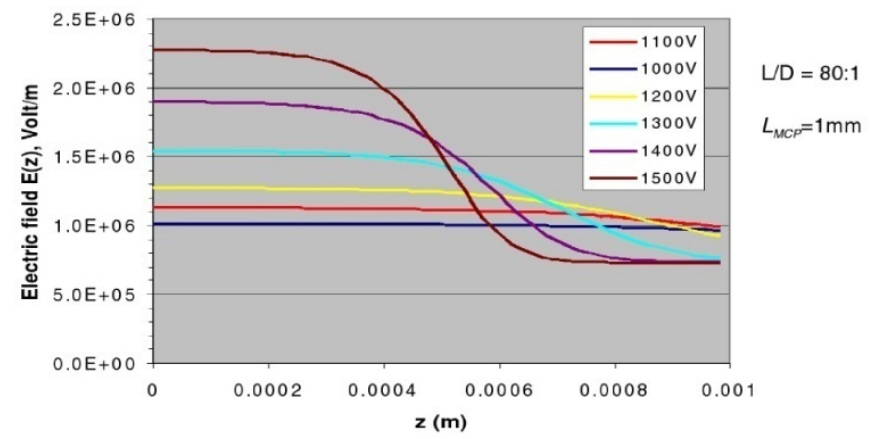

Figure $6 \mathrm{~b}$. The voltage along the channel. These predictions match the data well, giving us confidence in our understanding of the operation of MCP and the field configuration even in the presence of saturation (from A. Tremsin $[30])$.

\section{Capillary Glass Channel Substrate.}

We are investigating another attractive solution for the amplification substrate using conventional technology [53]. Glass capillary plates are available with $20-40 \mu \mathrm{m}$ channels, an aspect ratio of 40:1, and a bias angle of 8 degrees

solving the first-strike problem. Presently available small pore plates have open area ratios of $65 \%$, with $83 \%$ available for larger pore sizes.

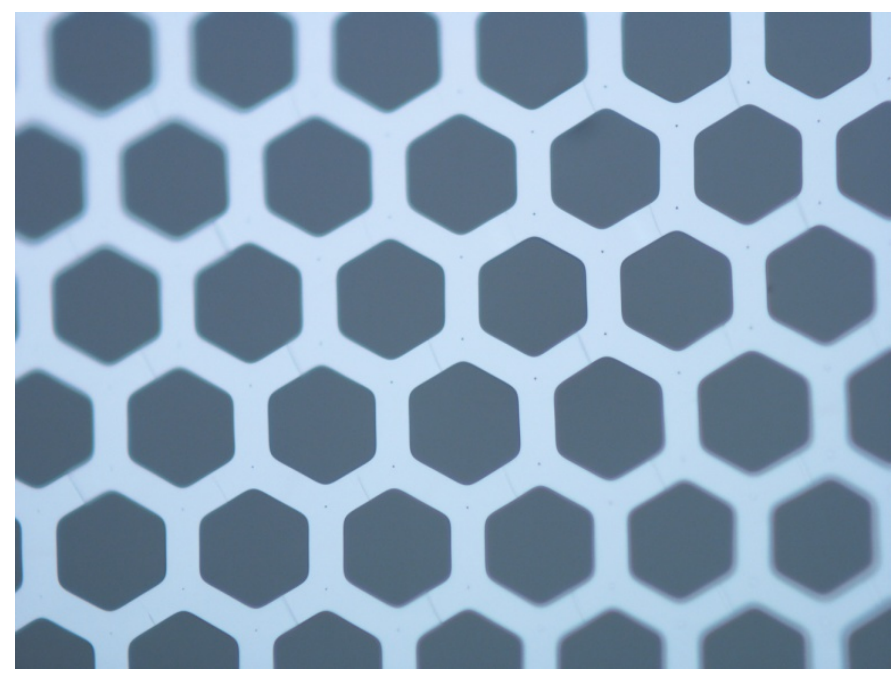

Figure 7. A 500x-magnification microscope image of a capillary array with $65 \%$ open area from Incom Inc. A microscope image of a glass 40 -micron diameter

Figure 7 shows an array of 40-micron diameter capillaries with an open area ratio of $65 \%$. The left-hand panel of Figure 8 shows a substrate with 50 micron pores. Development is underway to reduce the available pore size to $10 \mu \mathrm{m}$ or below (see the right-hand panel of Figure 8 [54]).

The capillary glass plates we are considering are much simpler to produce than those used in commercial MCP's, and consequently are much cheaper to produce in large areas. Because the resistive and amplification layers are implemented by functionalizing with ALD rather than the many-step reducing treatment used in conventional MCP production, these substrates provide only the physical substrate geometry, with no special requirements on the type of glass or the surface treatment.

In addition, the type of glass used is decoupled from the amplification requirements, so that inexpensive and robust boro-silicate glasses able to withstand temperatures well above $700 \mathrm{C}$ (even up beyond $1500 \mathrm{C}$ ) can be used [48].

We are currently aggressively pursuing this path with 32.8 $\mathrm{mm}$ samples to test the ALD and photocathode characterization. This path may prove to be a more-thanacceptable solution that is available now. The use of glass capillary substrates would also allow us to go ahead more quickly on the ALD optimization. 


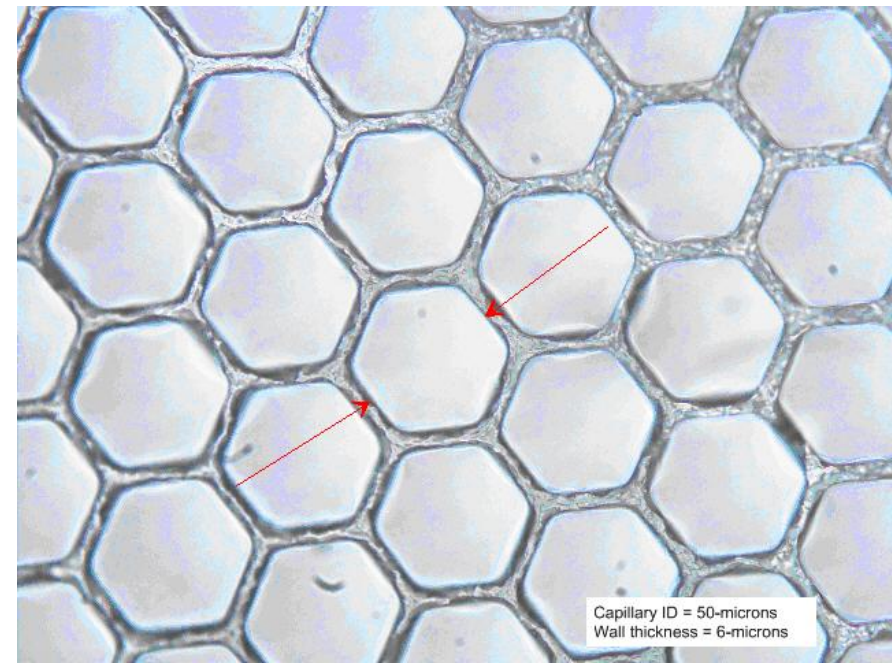

Figure 8a. A 500x-magnification microscope image of a capillary array from Incom Inc. Wafer dimensions are 1-inch $x 1$-inch by 0.1 -inch thick. The capillary internal diameter measured flat-to-flat is $50 \mu \mathrm{m}$, with a wall thickness of $6 \mu \mathrm{m}$.

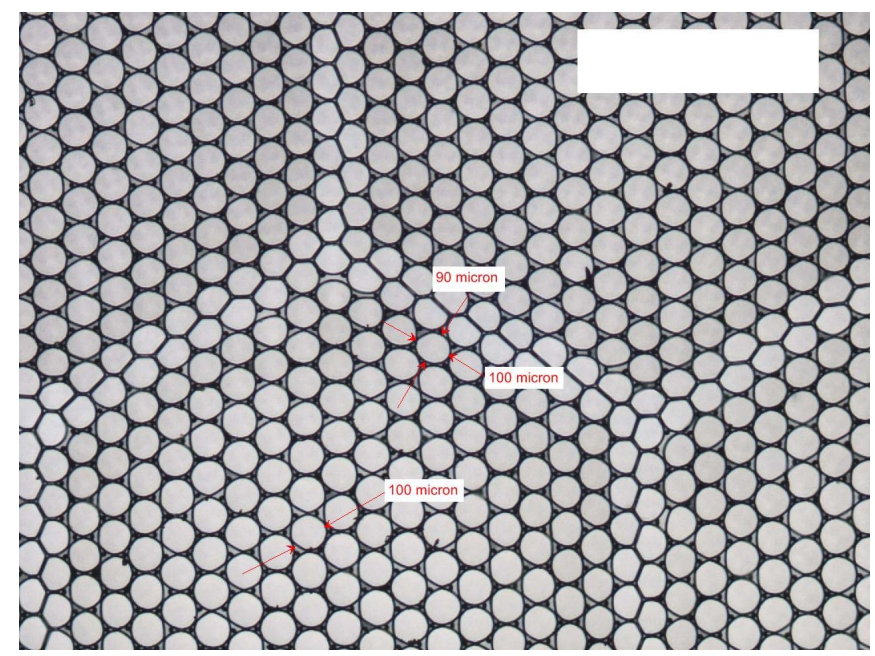

Figure 8b. A 500x-magnification microscope image of a MCP array from Incom Inc. with $10-\mu \mathrm{m}$ diameter channels, measured flat-to-flat. The wafer is 2 -inch $\times 2$-inch by 0.055 -inch thick.

We thus regard the capillary glass development path as the most-likely path to low-cost substrates.

However we are also considering several more radical alternatives that have some attractive characteristics and that may lead to new applications or developments, and these are discussed below

\section{Self-Organized Channel Substrates.}

Self-Organized substrates that can be produced in batch processes are attractive as possible economical alternatives to glasses. These alternative substrates may allow different capabilities, such as low background radiation compared to glass, or different geometries such as funnel entrances for opaque photo-cathodes. We plan on exploring these novel alternatives to glass capillaries until we understand the best solution for cost and mechanical properties.

One such substrate is Anodic Aluminum Oxide, AAO. AAO is a well-known self-organized material that contains highly uniform cylindrical nanopores, and can be made in a batch process. However, pores made in an intrinsic process (i.e. not micro-machined) are naturally small, typically a few hundred nanometers. To make MCP channels, pore diameters in intrinsic AAO of at least $>0.5 \mu \mathrm{m}$, and preferably $>1 \mu \mathrm{m}$, are needed.

Synkera has demonstrated the feasibility of 0.5-0.6 $\mu \mathrm{m}$ channels with a funnel-like entrance on one face, and complementary research is going on at Argonne in the Materials Science Division.

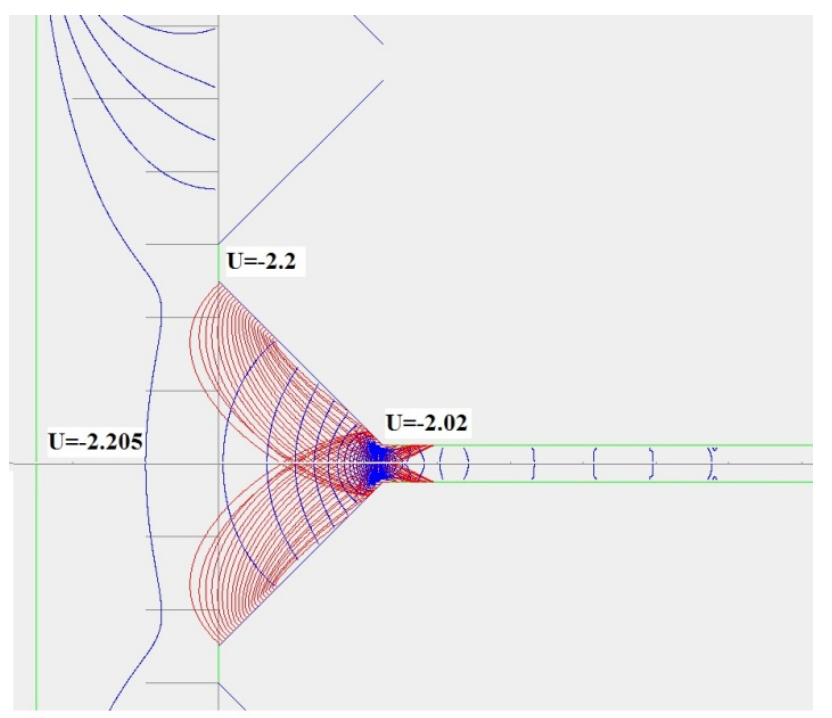

Figure 9a. A sketch showing the funnel solution to the first-strike problem. The funnel surface would be coated with a secondary emitter, either the photo-cathode or a first-strike surface (in the first case the window would be coated with a transparent conducting surface optimized for non-reflection; in the second the window coating would be the photo-cathode). By moving the photocathode out of the line of the channel one ameliorates the ion-aging problem, as well as possibly allowing for smaller (e.g. $1 \mu \mathrm{m})$ channels with a large fractional coverage.
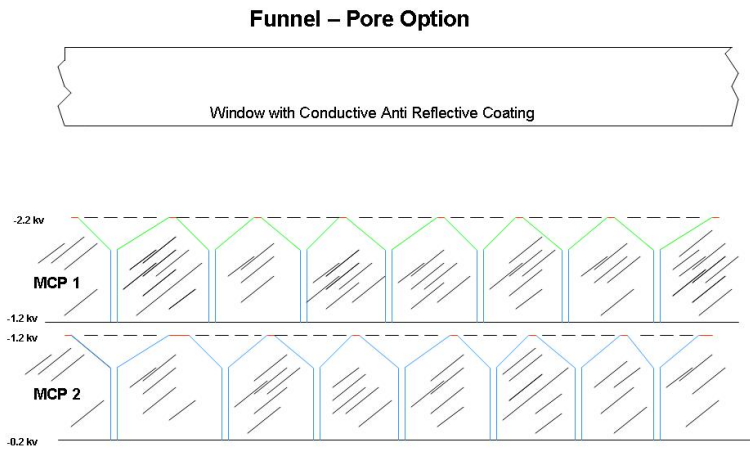

Figure 9b. A simulation [55] of the equi-potentials (blue) and secondary electron trajectories (red) for a small-channel/large-open-area AAO design (the top window is on the left, and the channel runs to the right.) 
We have several proposals for improving the open-area ratio and better determining the place of first strike, including making the entrance to the channel into a funnel, as shown in Figure 9a, so that the electron strikes the surface of the funnel and secondary electrons are sucked into the channel. Alternatively, the funnel could be directly coated with the photo-cathode material, with the photoelectron or electrons then initiating the shower in the channel, as shown in the simulation Figure 9b. The funnel solution is also attractive in that it hides the photo-cathode from ion feedback ions that are created on the channel walls and accelerated back up the channel [56-57]. These ions are a cause ofaging of MCP's, and can be a problem at high gain.

The development of ALD and AAO will be done in the small 'development' format, which is $32.8 \mathrm{~mm}$ round, for speed and ease of testing. Static testing will be done at Argonne, Arradiance, SSL, and Synkera.

\section{E. Aerogel Substrates.}

While the traditional architecture for electron amplifying channel plates has been arrays of micron sized tubes, the only scaffold requirements are 1) a high surface area of electron amplifying medium to provide gain, 2) a strong electric field gradient to guide electrons and recharge the surface, and 3) a large open porosity to allow electrons to traverse from front to back. Aerogels are relatively inexpensive materials that when functionalized by Atomic Layer Deposition can also provide these three requirements. When compared to conventional channel plates, aerogel-templated materials have larger open area ratios, larger surface areas, and are relatively easy to fabricate in large flat form factors. We have already demonstrated that aerogels can both be functionalized and work well as photo-anodes for solar cells [58-69].

The on-going program involving Argonne on aerogels can be used to prepare samples for characterization and gain measurements with little additional investment of effort or funding.

\section{F. Testing of the Functionalized Substrates}

The dynamic response of the development MCP plates will be tested using an X-ray streak camera now in operation at Sector 7 of the APS. In this device, electrons are created by a laser striking a photocathode, and then focused on the sample MCP. The output pulse from the MCP is then streaked out to precisely determine its waveform. The current leaving the sample MCP may also be measured electronically by steering it statically onto an optional microwave strip line near the readout $\mathrm{MCP}$, providing a measurement of the gain.

Facilities for the simpler static tests of gain can be performed at Arradiance, Synkera, SSL, and in multiple setups at Argonne.

\section{G. The Charge Collection and Digitization Problem}

In order to take advantage of large-area photo-detectors with good intrinsic space and time resolution, one has to solve the problems of building high-speed front-end electronics systems with low power and cost, and also collecting signal over distances large compared to the time resolution while preserving the fast time resolution inherent in the small feature size of the detectors themselves. Since some of the applications such as large water Cherenkov detectors require thousands of square meters of photo-detector, the readout electronics have to be integrated with the photo-detector itself in order to provide adequate analog band-width and to reduce the channel count and power. The two problems require an integrated solution.

The physical design proposed here uses strip transmission lines [42] which serve as the MCP anodes, i.e. are inside the vacuum volume. Both ends of each line are sampled by a channel of the front-end waveform sampling chip. The time of a pulse is given by the average of the times measured at the two ends, and the position along the line by the difference, as shown in Figure 13. The orthogonal coordinate is given by which lines are hit and the relative pulse heights and shapes. Simulations of the integrated system for a 2-inch-square MCP give expected time resolutions down to several picoseconds and spatial resolutions of $0.1 \mathrm{~mm}$ [6]. We have recently made the corresponding measurements, shown in Figure 12, and find a spatial resolution of $97 \mu \mathrm{m}$ corresponding to a relative time resolution between the two ends of several picoseconds which are also in excellent agreement with the simulation.

Simulations of 48-inch-long transmission lines, such as one might have in a large water Cherenkov detector at DUSEL, show the bandwidth remains above $1 \mathrm{GHz}$, as shown in Figure 13.

On should note that space and time resolution will probably be necessary in low-cost large-panel detector technologies as the noise rate from an emitter-based cathode or a batchprocess MCP may integrate over large areas to be significant.

Spatial resolution allows the identification of 'hot-spots' which then can be identified and eliminated in the local FPGA without ever entering the data stream. This is particularly important given our goal of very high QE photo-cathodes, which well may have locally high dark-currents due to occasional hot-spots of field-emission due to surface defects or impurities. We can suppress these in the front-end electronics automatically, with the FPGA itself keeping only an internal list of the areas that are bad actors. This would not be feasible while maintaining high efficiency without superb space and time resolution.

The measurements results with this transmission lines readout are presented at this Conference, see "Position Sensing using Pico-Second Timing with MicroChannel Plate Devices and Waveform Sampling". A test using a calibrated laser source [59] provided space resolutions on the order of a few hundreds of $\mu \mathrm{m}$ (Figure 12). The combined solution of transmission lines with waveform sampling at each end allows covering large areas with a 1-dimensional array, with a greatly reduced channel count and expense. The system provides excellent temporal and 2-dimensional spatial resolution as an added bonus intrinsic to the design. 


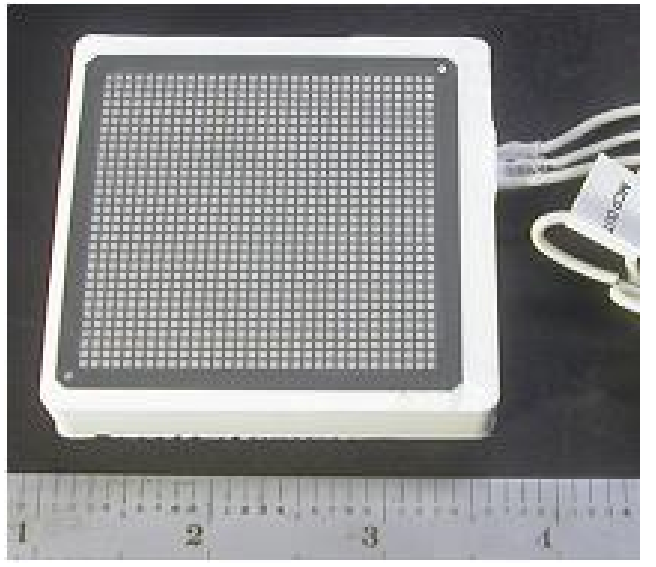

Figure 10a. The $32 \times 32$ array of anode pads on the Photonis MCP we are presently using to test the simulation and the transmission line boards.

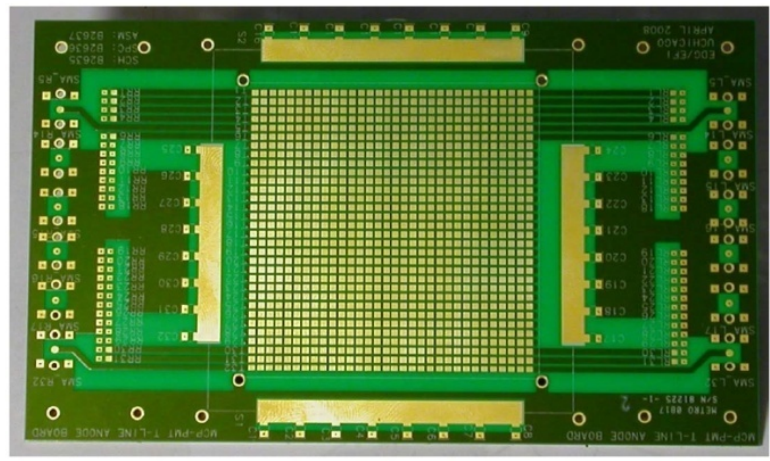

Figure $10 \mathrm{~b}$. The layout of the transmission line board showing the $32 \times 32$ array of pads which connect to the anode pads on the MCP. In the proposed devices the transmission lines will be inside the vacuum enclosure and will form the anodes directly, a simpler and more robust solution

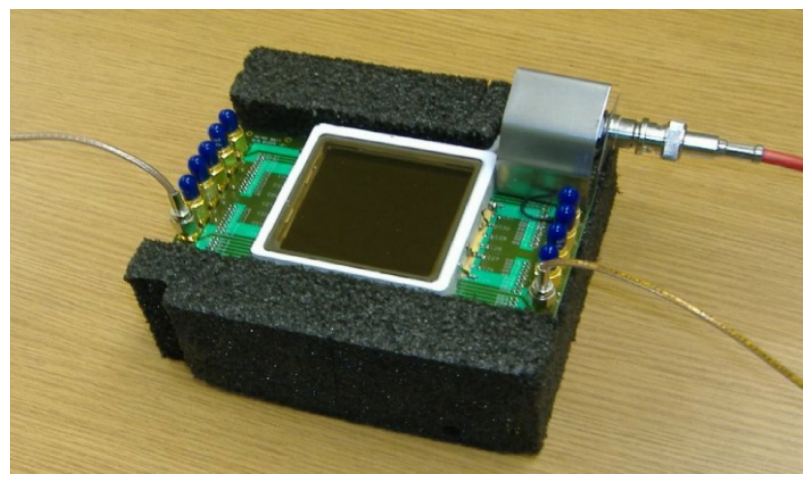

Figure 11a. The Photonis and transmission line readout anode card.

\section{System IsSuES: REAdout, CALIBRATION, AND CLOCK DISTRIBUTION}

The sampling chips require a non-trivial infrastructure to be a system. The Argonne HEP electronics engineering group has put substantial effort into investigating a clock distribution system with pico-second stability and control, and have a demonstration board that meets our initial specs. Preliminary designs of the interface to a XILINX FPGA to control and readout the front-end chips also exist.

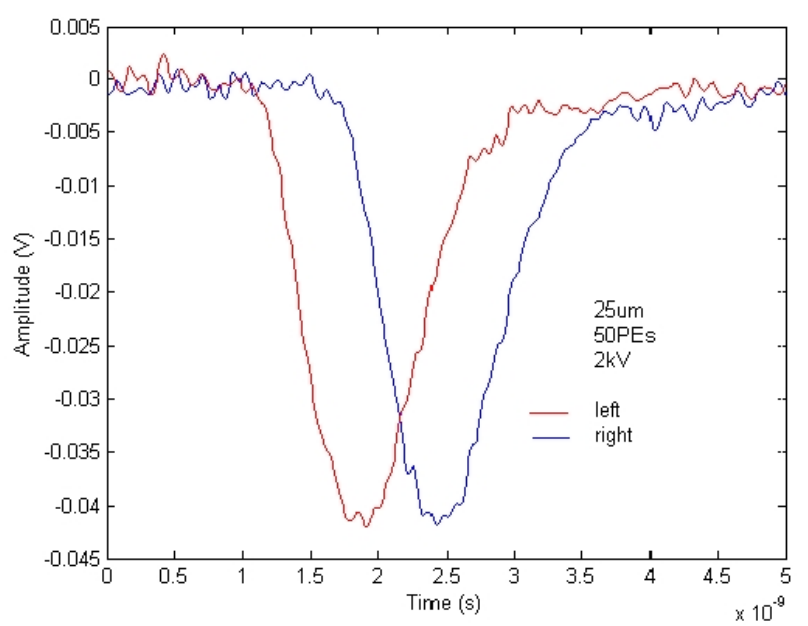

Figure 11b. A scope trace of the two ends of a transmission line for a laser pulse equivalent to 50 incident photo-electrons at the MCP entrance.

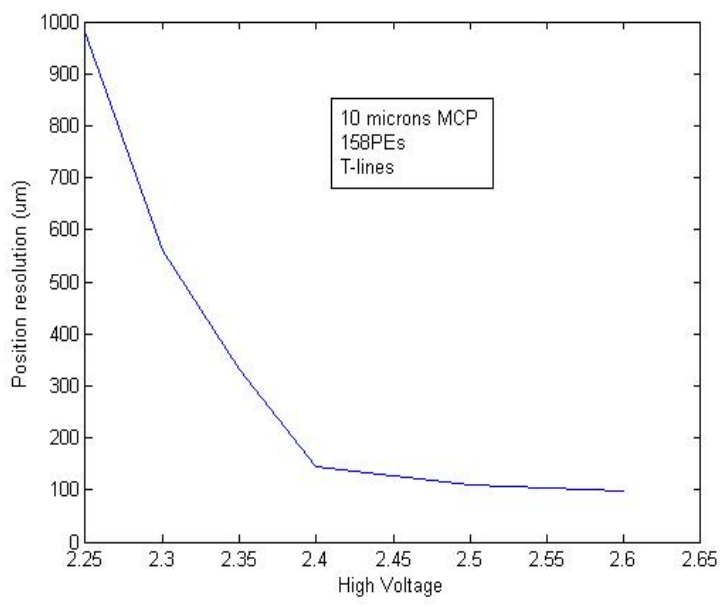

Figure 12. Measurements of the MCP/transmission-line readout taken with the Argonne fast laser tests tand, showing the spatial resolution versus signal size. At $2.6 \mathrm{KV}$ the measured resolution is $97 \mu \mathrm{m}$ with a $10-\mu \mathrm{m}$ pores MCP.

\section{MECHANICAL ASSEMBLY}

\section{A. Scaling Conventional Ceramic Technology}

To get to larger size individual MCP's, an extension of the ceramic technology that is used in space exploration and night vision to larger detectors will be started at SSL. This is a proven technology that has high reliability and low cost, and is used by night vision vendors to provide assemblies at thousands of units per month wit high reliability and low cost. Figure 14 shows the mechanical assembly for the ceramic technology used by SSL.

The costs for large-area are dominated by other factors than readout; the differential savings in eliminating spatial resolution we believe to be small while greatly increasing the risk and diminishing the capabilities. The scale-up of the ceramic technology to the 8-inch $\mathrm{x} 8$-inch module is relatively straight-forward, with a well-known set of possible problem areas: indium seal success ratios, design and braze success rates, window costs, and materials selection. 


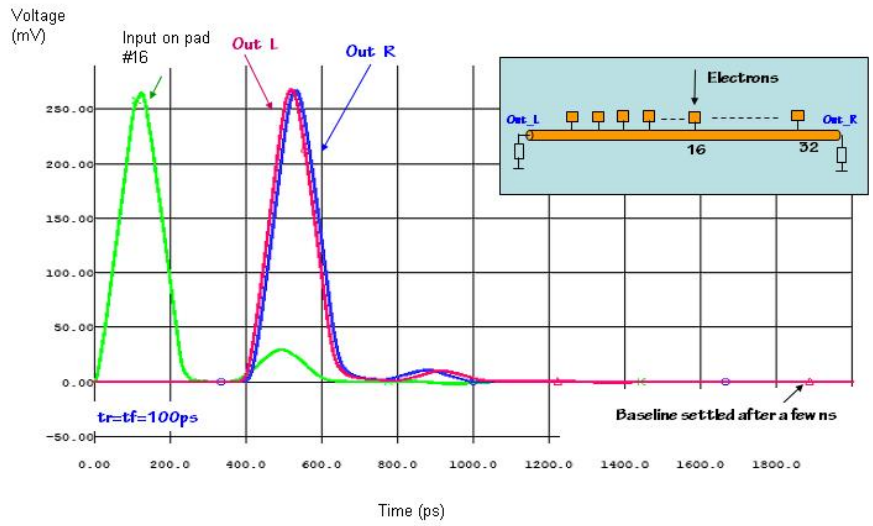

Figure 13a. A SPICE simulation of a linear model of the transmission line, extracted from the physical parameters of the printed-circuit card of Figure 10.

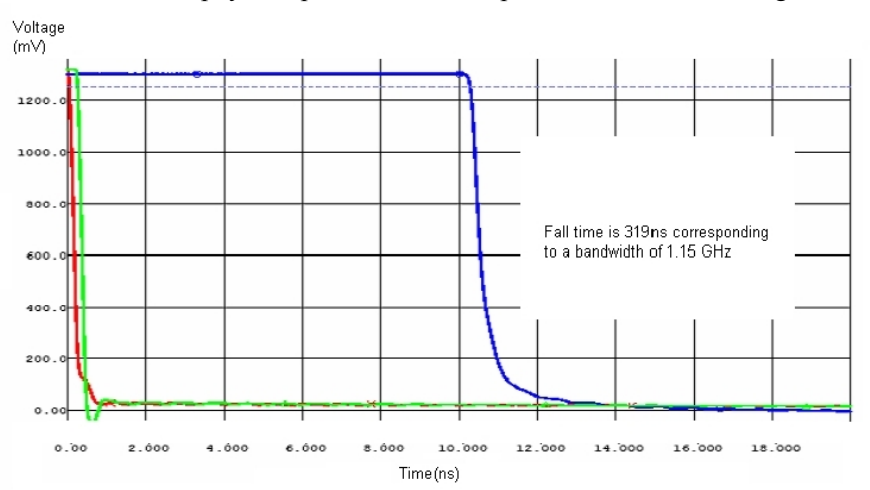

Figure 13b. The same simulation for a 48-inch long transmission line. We find an analog bandwidth of $1.1 \mathrm{GHz}$, more than adequate for very large area applications such as a water Cherenkov counter for DUSEL. The performance of the 40-inch x 20-inch module proposed here should be better than this result, as the anode lines are inside the vacuum, and there will be no'stubs' connecting the line to the MCP.

The larger alumina anodes should not be a problem provided that the strip anode and ground planes are properly applied, the braze materials are chosen correctly, and the process is shaken down.

Hydrogen contamination will be avoided by using vacuum brazing, in which LBNL has expert capabilities. We regard this path, which satisfies several of the highest priority applications, as low risk.

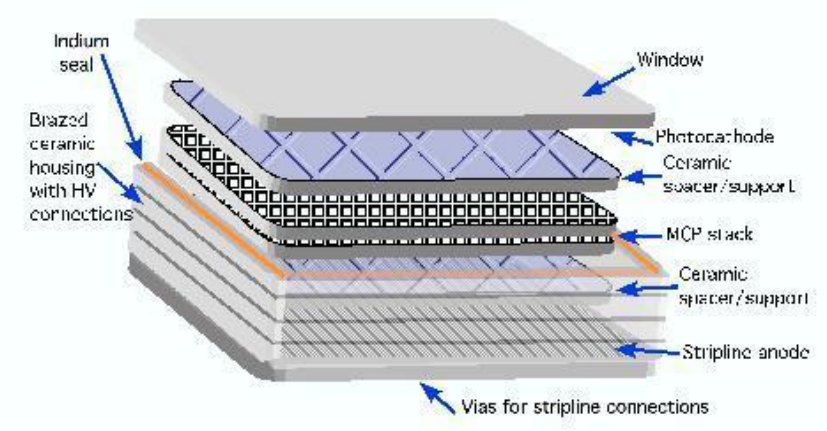

Figure 14a. The construction of a ceramic MCP-PMT.

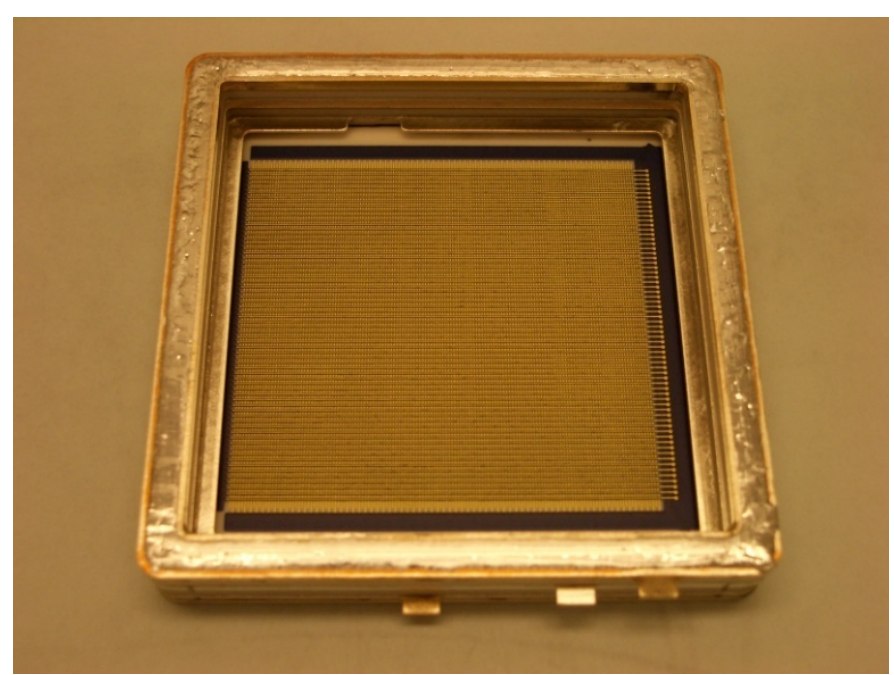

Figure 14b. A photograph of the mechanical assembly, showing the ceramic-substrate anode plane that forms an integral stack with ceramic spacers.

\section{B. All-Glass Detector Modules}

However, the mechanical assembly of very large-area photo-detectors, such as the $4^{\prime} \times 2^{\prime}$ panel, is a major challenge.

There are issues of mechanical registration and connection of the internal planes to form a monolithic package, electrical connections both internal and external, vacuum transfer, and assembly. An assembly facility will need to provide the ability to process and seal the advanced cathodes, MCPs and the front face in vacuum and bake and hot-scrub the MCPs.

We will try to exploit advances in flat-panel and solar energy technology to make a flat all-glass design. The design would be based on thin glass technology with laser-cutting and welding; pressure resistance would be provided by glass lattice spacers, much as load-bearing walls in a house provide pressure transfer from top to bottom. We are investigating the use of glass for the anode transmission lines as well; a range of dielectric constants with acceptable loss angles (determined from our simulations) is available.

Among the many concerns we are addressing are the mechanisms for making the internal layers into an integral amplification section, electrical charging of non-emitting surfaces, electrical connections and penetrations, and mechanical stability and robustness.

\section{DESIGNS.}

We plan to make as deliverables 3 separate designs of planar photo-detectors:

1. A $32.8 \mathrm{~mm}$ round format micro-channel plate, the 'Development' size, which we use as a test bed for characterizing new photo-emitting and secondary emitting materials. In addition to using the bare plates for characterization, this is a standard size used by SSL, and so we 
already have designs and parts for mechanical and electrical assembly to test the complete chain from photo-cathode to output, as shown in Figure 15.

2. A square glass-body 'frugal' 8-inch x 8-inch MCP-PMT, schematically shown in Figure 1. The 8-inch size of the photocathode, amplification section, and anode forms the subunit out of which larger devices can be assembled. The assembled module is also a stand alone device that replace a 10 -inch tube, base, and front-end electronics assembly.

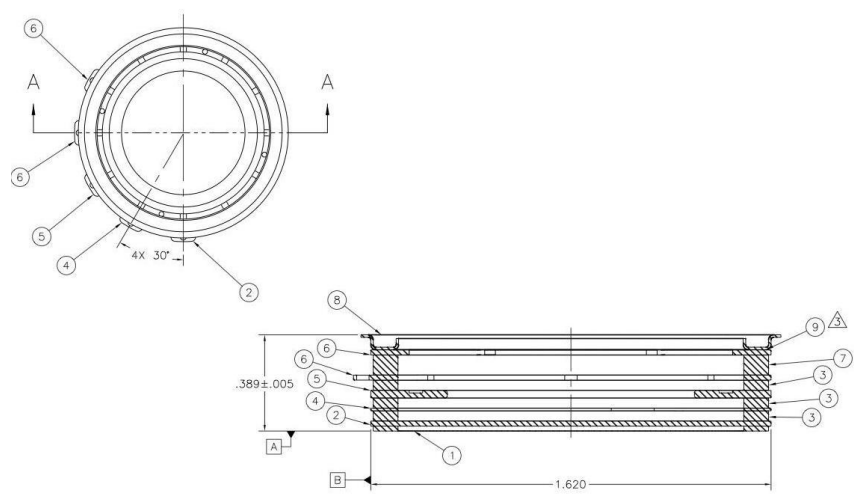

Figure 15a. The existing mechanical design for the Development $32.8 \mathrm{~mm}$ MCP-PMT, used for rapid photo-cathode and secondary emission development and characterization of materials.

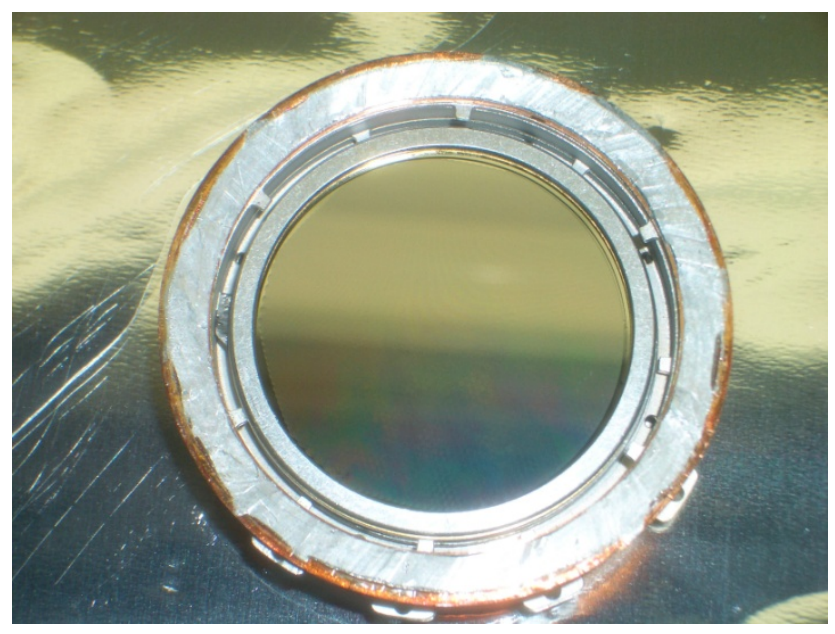

Figure 15b. An existing MCP-PMT in the Development format.

3. A 4-inch $\times 2$-inch panel consisting of multiple 8-inch $\times 8$ inch photo-cathodes and amplification sections, feeding a continuous common anode with 4-foot long strip transmission lines, as shown in Figure 16 (also see Figure 13 for a simulation of the anode performance). This is the proto-type for DUSEL and other very large-area applications.

We have settled on 8-inch $\times$ 8-inch from initial studies; it may be that a somewhat larger size (e.g. 12-inch square) will turn out to be optimum in terms of cost of tooling and assembly, but the principle and basic details remain the same.

\section{SUMMARY}

While what we are proposing is not easy, there are demonstrated solutions to each of the individual pieces.

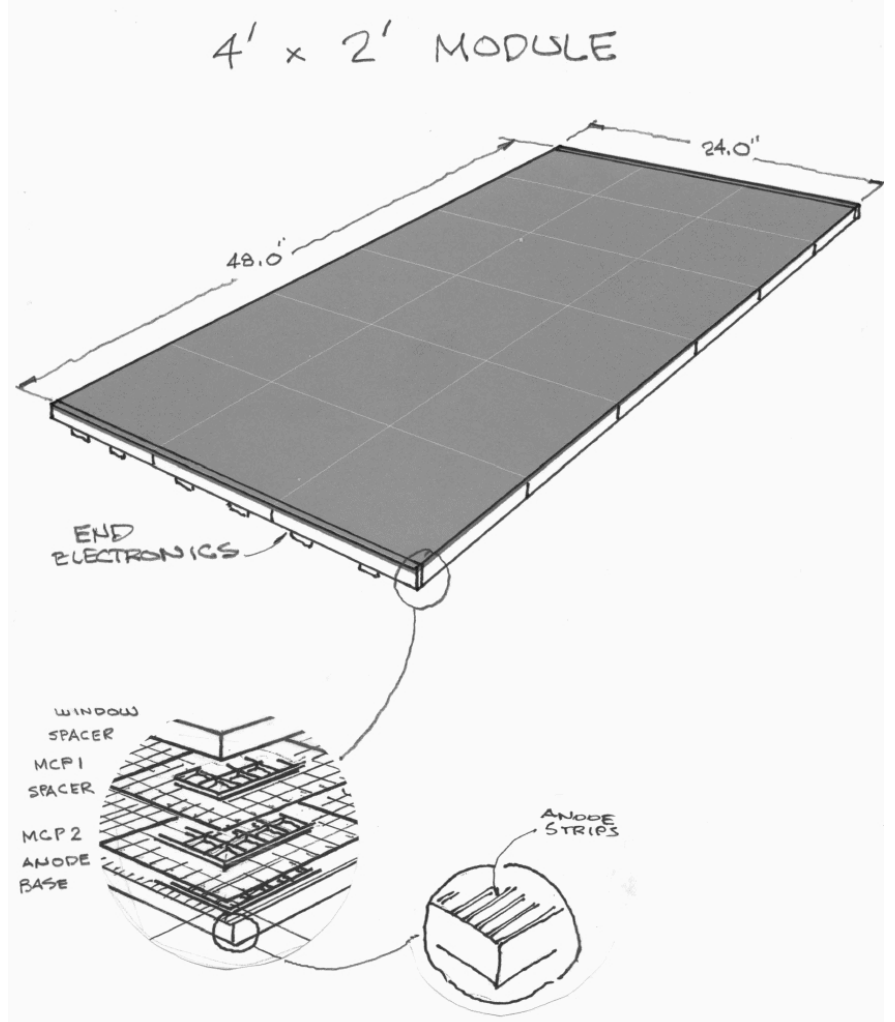

Figure 16. The 4-foot x 2-foot panel, with a mosaic of photo-cathodes and AAO amplification plates, and a single anode consisting of 4-foot long transmission lines. Simulation results give an analog bandwidth of $1.1 \mathrm{GHz}$ for the readout, more than adequate for DUSEL.

Readout of MCP-PMTs by transmission-line anodes with wave-form sampling on both ends has been demonstrated and a detailed simulation that predicts good performance for modules up to 4-feet long has been validated by measurements. Industry routinely makes bialkali photocathodes for PMTs and MCP-PMTs; we need to improve the quantum-efficiency of the SSL photo-cathodes from $20 \%$ up to typical industry values and scale up in size, and to solve the problems of mechanical assembly into large panels. Glass capillary substrates look very promising as an available and economical MCP substrate, with several nano-material alternatives under investigation. The superior performance of ALD-coated glass substrate MCP's has been demonstrated, and ALD is already used in industry at the scales we need. The problems of mechanical assembly of scale-up to large (e.g. 4foot $x$ 2-foot ) panels are formidable, as they involve in situ photo-cathode production, ultra-high-vacuum transfer, bakeout and scrubbing, glass-to-glass and glass-to-metal seals, surface physics issues (e.g. dark-current, non-channel noise sources, and aging), ASIC design, electronics systems design, and integration. However we do not see any show-stoppers; just a lot of hard work. In parallel we will pursue synergistic 
programs of higher risk but very high pay-off to improve the single photo-electron energy resolution and the transit-time spread by optimizing the photo-cathode/channel geometry, and to improve photo-cathode quantum efficiencies using the expertise at Argonne in nano-scale materials, our advanced MCP simulations, and our facilities and experience for ALD.

\section{ACKNOWLEDGEMENT}

We gratefully thank Alan Bross (Fermilab), Joseph Gregar (Argonne) Paul Hink (Photonis), Michael Minot (Incom), and Bill Moses (LBNL) for helpful comments, criticisms, and suggestions in the preparation of this contribution.

\section{REFERENCES}

[1] J.W. Elam, G. Xiong, C.Y. Han, H.H Wang, J.P. Birrell, U. Welp, J.N. Hyrn, M.J. Pellin, T.F. Baumann, J.F. Poco, and J.H. Satcher, "Atomic Layer Deposition for the Conformal Coating of Nanoporous Materials", Journal of Nanomaterials, 2006, pp 1-5.

[2] J.W. Elam, J. A. Libera, M.J. Pellin, and P.C. Stair, "Spatially Controlled Atomic Layer Deposition in Porous Materials", Applied Physics Letters, 2007 $91(24)$

[3] http://www.synkera.com including the links to "Self-organized Anodic Aluminum Oxide and Ceramic MEMS" sections.

[4] http://www.industrial-lasers.com/display article/330518/39/none/none/eat/Laserglass-cutting-in-flat-panel-displayproduction

[5] J-F. Genat, G. Varner, F. Tang, H.J. Frisch, “ Signal Processing for Picosecond Resolution Timing Measurements" Submitted to Nucl. Inst. Meth

[6] J-F. Genat, K. Byrum, H.J. Frisch, M. Heintz, E.N. May, T. Natoly, F.Tang, E. Yurtsev, "Position Sensing using Pico-Second Timing withMicroChannel Plate Devices and Waveform Sampling". This Workshop.

[7] http://jp.hamamatsu.com/resources/products/etd/eng/html/pmt 003.html The Hamamatsu Ultra Bialkali photo-cathode has achieved a quantum efficiency of $43 \%$.

[8] www.hamamatsu.com For example, the Hamamatsu R9800 25-mm tube has a transit time spread (TTS) of 270 psec (FWHM).

\section{[9] http://sales.hamamatsu.com/multianode}

[10] K. Arisaka et al., "Performance of a Prototype Aerogel Counter Readout" http://www.scientificcommons.org/40872582 (2008)

[11] K. Arisaka et al., XAX: a multi-ton, multi-target detection system for dark matter, double beta decay and solar neutrinos, ArXiv http://arxiv.org/abs/0808.3968 (2008)

[12] Photonis/Burle Industries, 1000 New Holland Ave., Lancaster PA, 17601 http://www.photonis.com/industryscience/products/microchannel

[13] \http://sales.hamamatsu.com/en/products/electron-tubedivision/detectors/microchannel-platesmcps

$[14] \backslash \backslash$ http://www.photek.com
[15] O.H.W. Siegmund, Barry Welsh, J. Vallerga, A. Tremsin, J. McPhate, "High-performance microchannel plate imaging photon counters for spaceborne sensing", Proc. SPIE Vol. 6220 (2006) 622004

[16] O. H.V. Siegmund, J. Vallerga, P. Jelinsky,, X. Michalet, S. Weiss, "Cross delay line detectors for high time resolution astronomical polarimetry and biological fluorescence imaging", Nuclear Science Symposium, 2005 IEEE, Volume 1, 23-29 Oct. 2005 Page(s):448 - 452

[17] P. Jelinsky, P. Morrissey, J. Malloy, S. Jelinsky, and O. Siegmund, C. Martin, D. Schiminovich, K. Forster, T. Wyder and P. Friedman, Performance Results of the GALEX cross delay line detectors, Proc. SPIE Vol.4854, pp 233-240, 2003\} \bibitem \{siegmund4\} In addition to the above references, see:

[18] O.H.W. Siegmund, J. Vallerga, A. Tremsin, "Characterizations of microchannel plate quantum efficiency", Proc. SPIE Vol. 5898 (2005) $58980 \mathrm{H}\}$

[19] O. H. W. Siegmund, A. S. Tremsin, C. P. Beetz, Jr., R. W. Boerstler, D. R. Winn, "Progress on development of silicon micro-channel plates", SPIE Proc., vol. 4497, 139-148, 2002.

[20] O.H.W. Siegmund, J. Vallerga, and P. Lammert, "High amplitude events in Micro-channel Plates”, IEEE Trans. Nucl. Sci., NS-36, 830-835 (1989).

[21] V. Ivanov, "Computation Methods and Device Optimization in Electron Optics" (2 volumes, in Russian), Moscow, 2005.

[22] http://www.arradiance.com ,including a link to A. S. Tremsin, H. F. Lockwood, D. R. Beaulieu, N. T. Sullivan, E. Munro, J. Rouse, "3D microscopic model of electron amplification in micro-channel amplifiers for maskless lithography, 7th International Conference on Charged Particle Optics, Cambridge, England, July 2006, Physics Procedia 1 (2008) 565.

[23] A.B. Berkin, V.V. Vasilyev, "Numerical simulation of the amplification regime for pulsed current in the channel of MCP", June 2007 (in Russian), A.B. Berkin, V.V. Vasilyev, "Numerical model of the amplification regime for the direct current in the channel of MCP", June 2007 (in Russian), A.J. Guest, A computer model of channel multiplier plate performance, 1971.

[24] J. Milnes and J. Howorth, (Photek Ltd.), "Picosecond Time Response Characteristics of Micro-channel Plate PMT Detectors; SPIE USE V.8, 5880 (2004).

[25] D. Breton, E. Auge, E. Delagnes, J. Parsons, W Sippach, V. Tocut, "The HAMAC rad-hard Switched Capacitor Array". ATLAS note. October 2001.

[26] E. Delagnes, Y. Degerli, P. Goret, P. Nayman, F. Toussenel, and P. Vincent. "SAM : A new GHz sampling ASIC for the HESS-II Front-End", Cerenkov 2005

[27] S. Ritt. "The DRS chip: A Cheap waveform digitizing in the GHz range" Nucl.Instrum.Meth.A518:470-471, 2004.

[28] G. Varner, L.L. Ruckman, J.W. Nam, R.J. Nichol, J. Cao, P.W. Gorham, and M. Wilcox. "The Large Analog Bandwidth Recorder And Digitizer with Ordered Readout (LABRADOR) ASIC", Nucl.Instrum.Meth. A583:447-460, 2007

[29] J.-F. Genat, G. Varner, F. Tang, H. Frisch; "Signal Processing for Picosecond Resolution Timing Measurements", To be published in Nucl. Instr. Meth., 2009; arXiv:0810.5590. We have noticed in our discussions with groups interested in applications that our natural translation of time resolution into space resolution along the distance of travel is not commonly shared; it takes some getting used to for the words "time resolution" of $10 \mathrm{ps}$ to naturally represent a resolution of $3 \mathrm{~mm}$ (or less if in a medium) in the direction the photon or charged particle came from. 
[30] For the agendas and slides of the last 7 workshops, see http://psec.uchicago.edu/workshops.php

[31] Q. Xie, C.-M. Kao, X. Wang, N. Guo, C. Zhu, H. Frisch, W.W. Moses, C.-T. Chen; "Potential Advantages of Digitally Sampling Scintillation Pulses in Timing Determination in PET", Nuclear Science Symposium Conference Record, 2007. NSS 2007; Vol. 6: 4271-4274\}

[32] H. Kim C.-M. Kao, Q. Xie, H. Frisch, W. W. Moses, W.-S. Choong, L. Zhou, F. Tang, J. Lin, O. Biris, and C.-T. Chen; "A Multi-Threshold Method for TOF-PET Signal Processing”. Nucl. Instrum. Meth. A 602, 618-621 (2009).

[33] D. Herbst, "Time of Flight in PET Using Fast Timing and Leading Edge Fit Optimization", Submitted to IEEE08, October, 2008; Dresden Germany\}

[34] J. Lin, O. Biris, C.-T. Chen, W.-S. Choong, H. Frisch, C.-M. Kao, W. W. Moses, F. Tang, Q. Xie, L. Zhou; "Electronics development for fast-timing PET detectors: The multi-threshold discriminator Time of Flight PET system", Proceedings of SORMA, Berkeley CA, May, 2008.

[35] Q. Xie, C.-M. Kao, X. Wang, N. Guo, C. Zhu, H. Frisch, W.W. Moses, C.T. Chen; "Potential Advantages of Digitally Sampling Scintillation Pulses in Time Determination in PET", NSSC/MIC IEEE, Honolulu, Hawaii, Oct 27Nov 3, 2007

[36] H. Nicholson. Private communication. We are also grateful for the strong push toward developing much larger detector modules than we had envisioned

[37] We thank Neville Harnew for guidance on the LHCb requirements. A typical design for an LHCb upgrade is a coverage of $5 \mathrm{~m} \times 6 \mathrm{~m}$ (of order $10 \mathrm{~m}$ from the interaction point) to achieve charged-particle K- $\pi$ identification in the $1-10 \mathrm{GeV}$ range. The system easily fits in the available longitudinal length of $30 \mathrm{~cm}$.

[38] William Moses. Private communication. We thank Dr. Moses for also providing us with a rough cost estimate for a typical system.

[39] O. Siegmund, J. Vallerga, J. McPhate, J. Malloy, A. Tremsin, A. Martin, M. Ulmer and B. Wessels, "Development of GaN photocathodes for UV detectors", Nuclear Instruments and Methods in Physics Research Section A: Vol. 567, Issue 1, 2006, pp 89-92.

[40] A.S. Tremsin, O.H.W. Siegmund, "Quantum efficiency and stability of alkali halide UV photocathodes in the presence of electric field", Nuclear Instruments and Methods in Physics Research (sect. A), Vol. 504 (2003), pp 4-8.

[41] A.S. Tremsin and O.H.W. Siegmund, "Polycrystalline diamond films as prospective UV photocathodes", Proc. SPIE, 4139, 16, (2000).

[42] See, for just one example: http://www.industrial-lasers.com/display article/330518/39/none/none/eat/Laserglass-cutting-in-flat-panel-displayproduction. We have initiated contacts with a number of firms in the industry, and also have dedicated glass expertise on the staff at Argonne. We have started design work at Argonne and Chicago on the details of a glass solution, including the best way to connect to the HV and signals. What is shown is just a 'cartoon' illustrating the basic ideas.

[43] K. Attenkofer, Workshop on the Development of Large Area Psec Photo-Devices; Workshop VII, Argonne National Laboratory, Lemont, IL; Feb. 2009; http://hep.uchicago.edu/psec/

[44] H.J. Frisch, H. Sanders, F. Tang, and T. Credo, United States Patent 7485872, Large area, pico-second resolution, time of flight detectors

[45] Masaki Ishitsuka, " $\mathrm{L} / \mathrm{E}$ analysis of the atmospheric neutrino data from Super-Kamiokande"; Ph.D Thesis, University of Tokyo, Feb., 2004.

[46] We thank Paul Hink (Photonis) for influential input to this section.
[47] This is the term used to describe the coating of the substrate with emittive materials to provide amplification.

[48] The direction of the field lines in the channel is a function of the bulk and surface resistances, and has been the subject of some controversy. In the devices we consider here the lines are parallel to the channel axis provided one is more than several channel diameters away from the ends.

[49] R. R. Reagan. "Trust but validate", on signing the 1987 Intermediate Nuclear Forces treaty. http://www.usemod.com/cgi-bin/mb.pl TrustButVerify

[50] http://www.arradiance.com/, including a link to: D. R. Beaulieu, D Gorelikov, P. de Rouffignac, K. Saadatmand, K. Stenton, N. Sullivan, A. S. Tremsin; "Nano-engineered ultra high gain microchannel plates".

[51] J.W. Elam, D. Routkevitch, S. M. George, "Properties of $\mathrm{ZnO} / \mathrm{Al} 2 \mathrm{O} 3$ Allow Films Grown Using Atomic Layer Deposition Techniques", J. of the Electrochem. Soc 2003 150(6) G339

[52] J.W. Elam, J.T. Hupp, and M.J. Pellin, "ZnO Nanotube-Based DyeSensitized Solar Cells", NanoLetters, 2007(7), P.2183.

[53] A. Tremsin. Private communication.

[54] We thank Michael Minot (Incom) and Joseph Renaud from Incom.Inc for the pictures and information.

[55] V. Ivanov. "Computation Methods and Device Optimization in Electron Optics" (2 volumes, in Russian), Moscow, 2005.

[56] K. Inami, Workshop on Timing Detectors, Institute of Nuclear Physics in Lyon (IPNL) France, Oct. 2008.

[57] J. Va'Vra, D.W.G.S Leith, B. Ratcliff, E. Ramberg, M. Albrow, A. Ronzhin, C. Ertley, T. Natoli, E. May and K. Byrum, "Progress on the Development of a New TOF Concept using MCP-PMTs Detectors" submitted to Nucl. Instr. Meth., March 2009.

[58] Hamann, T. W., A. B. F. Martinson, et al. "Atomic Layer Deposition of $\mathrm{TiO} 2$ on Aerogel Templates for Novel Photoanodes in Dye-Sensitized Solar Cells". Journal of Physical Chemistry C 112(27): 10303-10307. 2008.

[59] Hamann, T. W., A. B. F. Martinson, et al. "Aerogel Templated ZnO DyeSensitized Solar Cells”. Advanced Materials 20(8): 1560-156 2008.

[60] E.N. May, To be published. 